\title{
Nintedanib plus docetaxel after progression on immune checkpoint inhibitor therapy: insights from VARGADO, a prospective study in patients with lung adenocarcinoma
}

\author{
Christian Grohé*,1, Wolfgang Gleiber ${ }^{2}$, Siegfried Haas ${ }^{3}$, Christoph Losem ${ }^{4}$, Harald \\ Mueller-Huesmann ${ }^{5}$, Mathias Schulze ${ }^{6}$, Christian Franke ${ }^{7}$, Nadezda Basara ${ }^{8}$, Judith Atz ${ }^{9}$ \& \\ Rolf Kaiser ${ }^{10,11}$ \\ ${ }^{1}$ Department of Respiratory Diseases, ELK, 13125, Berlin, Germany \\ ${ }^{2}$ University Hospital Frankfurt, Pneumology/Allergology, 60590 Frankfurt, Germany \\ ${ }^{3}$ Friedrich-Ebert Hospital Neumuenster, Clinics for Hematology, Oncology \& Nephrology, 24534 Neumuenster, Germany \\ ${ }^{4} \mathrm{MVZ}$ für Onkologie und Hämatologie im Rhein-Kreis Neuss, 41462 Neuss, Germany \\ ${ }^{5}$ Klinik für Hämatologie und Onkologie, Bruederkrankenhaus St Josef, 33098 Paderborn, Germany \\ ${ }^{6}$ Praxis Dr Schulze, 02763 Zittau, Germany \\ ${ }^{7}$ Facharztzentrum Sonneberg, 96515 Sonneberg, Germany \\ ${ }^{8}$ Department of Internal Medicine, Malteser Hospital, St Franziskus, 24939 Flensburg, Germany \\ ${ }^{9}$ Medical Affairs Oncology, Boehringer Ingelheim Pharma GmbH \& Co KG, 55216 Ingelheim Am Rhein, Germany \\ ${ }^{10}$ Boehringer Ingelheim Pharma GmbH \& Co, KG, 55216 Ingelheim Am Rhein, Germany \\ ${ }^{11}$ Institute of Pharmacology, Johannes Gutenberg-University Mainz, 55122 Mainz, Germany \\ *Author for correspondence: Christian.Grohe@pgdiakonie.de
}

\begin{abstract}
Aim: To assess outcomes in patients with advanced adenocarcinoma non-small-cell lung cancer who received nintedanib plus docetaxel after progression on prior chemotherapy followed by immune checkpoint inhibitor $(\mathrm{ICl})$ therapy. Patients \& methods: VARGADO is a prospective, noninterventional study. We describe initial data from a cohort of 22 patients who received nintedanib plus docetaxel after chemotherapy and $\mathrm{ICl}$ therapy. Results: Median progression-free survival with nintedanib plus docetaxel was 5.5 months $(95 \% \mathrm{Cl}: 1.9-8.7$ months). The objective response rate was $7 / 12(58 \%)$ and the disease control rate was $10 / 12$ (83\%). Data for overall survival rate 12 months after the start of treatment (primary end point) are not yet mature and are not reported. Of 22 patients, $73 \%$ experienced drug-related adverse events; adverse events led to treatment discontinuation in $32 \%$ of patients. Conclusion: These data highlight the potential clinical benefit of nintedanib plus docetaxel in patients who failed prior ICI therapy.
\end{abstract}

Trial registration number: NCT02392455

First draft submitted: 7 May 2019; Accepted for publication: 20 June 2019; Published online: 8 July 2019

Keywords: angio-immunogenic switch • docetaxel • nintedanib • non-small-cell lung cancer • tumor microenvironment • VARGADO

Nintedanib is an oral triple angiokinase inhibitor targeting VEGFRs $1-3$, PDGF receptors $\alpha / \beta$ and fibroblast growth factor receptors 1-3 [1], as well as RET [2]. Nintedanib is approved in the European Union and other countries in combination with docetaxel for the treatment of patients with locally advanced, metastatic or locally recurrent non-small-cell lung cancer (NSCLC) of adenocarcinoma histology after first-line chemotherapy [3]. This approval was based on data from the Phase III LUME-Lung 1 trial, in which nintedanib plus docetaxel was associated with a clinically meaningful extension in overall survival (OS) compared with placebo plus docetaxel in patients with adenocarcinoma NSCLC who had progressed after first-line chemotherapy [4].

Following the introduction of immune checkpoint inhibitors (ICIs), the treatment landscape in nontargetable advanced NSCLC has been changing dramatically [5]. With more, and better, treatment options now becoming available and many patients being treated with first- or second-line ICIs, a pressing question in clinical practice is, "what is the optimal treatment sequence after my patient has progressed on ICI \pm chemotherapy?" To answer

Future Medicine 


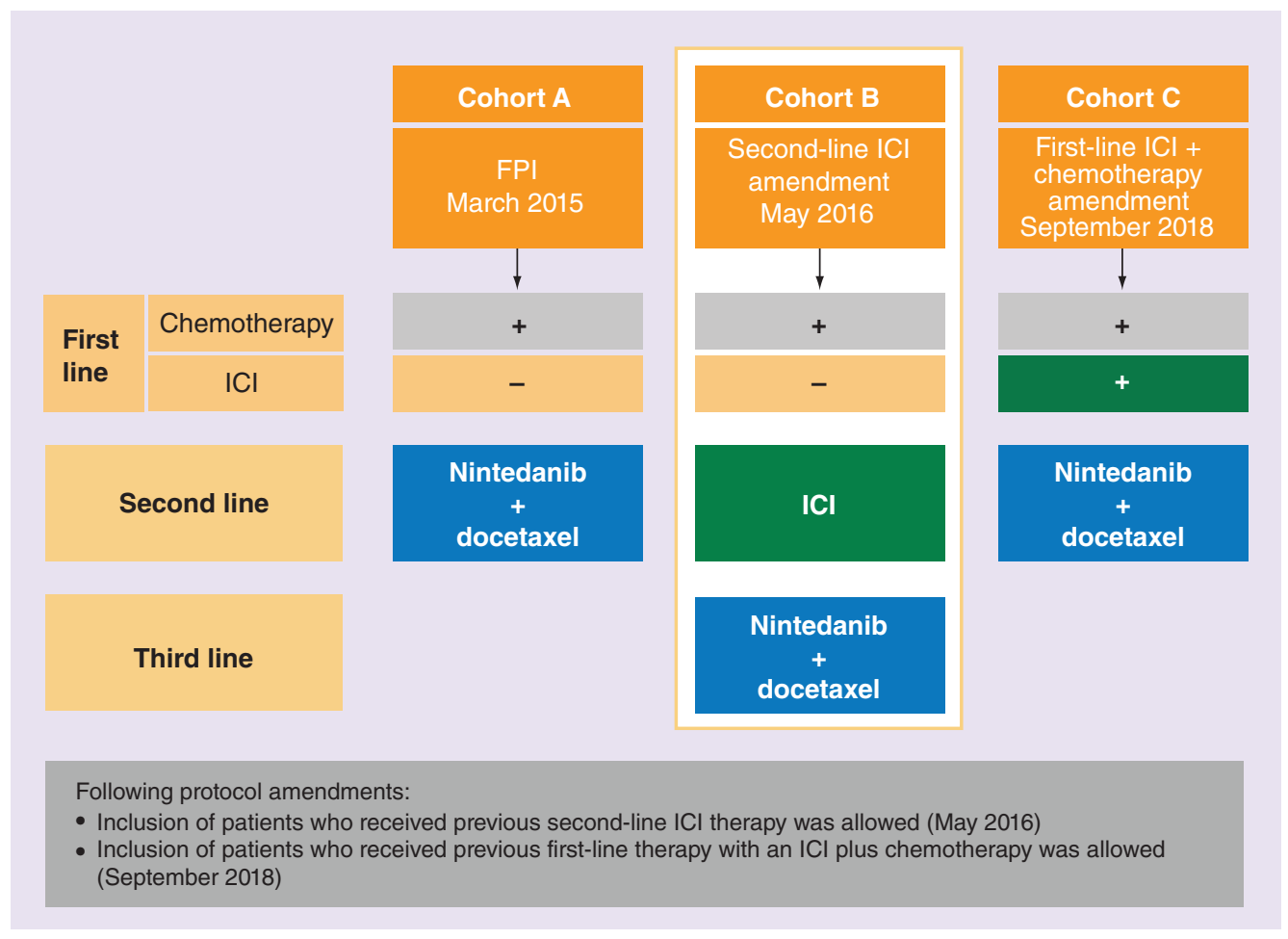

Figure 1. Patient cohorts in VARGADO.

FPI: First patient in; ICI: Immune checkpoint inhibitor.

this important clinical question, the underlying biology must also be considered, namely: how can we target the immunosuppressive tumor microenvironment (TME) in patients whose tumors have progressed on ICIs?

Considering the antiangiogenic treatment options in this patient population, there are very limited clinical data to help answer these questions. However, it has been shown that, in addition to its central role in angiogenesis, VEGF is involved in tumor-associated immunosuppression if it is present in excess in the TME [6]. Recently published results of a retrospective US study using electronic health record data showed that 161 patients who received the VEGFR2 antibody ramucirumab after previous ICI-based therapy had an unadjusted median OS of 26.5 months (95\% CI: 23.7-33.0) after diagnosis [7]. In another study, encouraging efficacy of later-line nintedanib combined with docetaxel was reported in 11 patients with adenocarcinoma NSCLC who had previously been treated with platinum-based doublet chemotherapy and immunotherapy: the response rate was $36.5 \%$ and the disease control rate (DCR) was $81.8 \%$ [8]. Here, we present data from a cohort of patients who received nintedanib plus docetaxel in this new clinical setting, in other words after chemotherapy and ICI therapy, in the noninterventional, prospective VARGADO study.

\section{Patients \& methods}

Patients \& study design

VARGADO is a prospective, noninterventional study of nintedanib plus docetaxel after first-line chemotherapy in the routine clinical treatment of patients with locally advanced, metastatic or locally recurrent adenocarcinoma NSCLC [9]. Three patient cohorts in VARGADO are being evaluated (Figure 1). The study is ongoing in 107 study sites across Germany.

Here, we describe an initial analysis of VARGADO cohort $\mathrm{B}$, in which 22 patients received nintedanib plus docetaxel after prior chemotherapy and treatment with an ICI: either nivolumab $(n=17)$ or pembrolizumab $(n=5)$.

Nintedanib and docetaxel were administered according to the approved label. Patients received docetaxel $\left(75 \mathrm{mg} / \mathrm{m}^{2}\right)$ by intravenous infusion on day 1 , plus oral nintedanib (200 $\mathrm{mg}$ twice daily) on days 2-21 of each 21-day cycle. Patients were followed-up for safety and efficacy for up to 24 months after the start of treatment. Data on patient characteristics, oncologic history, and prior and concomitant medication, as well as efficacy and safety data, were collected during routine clinic visits. All patients provided signed informed consent. 


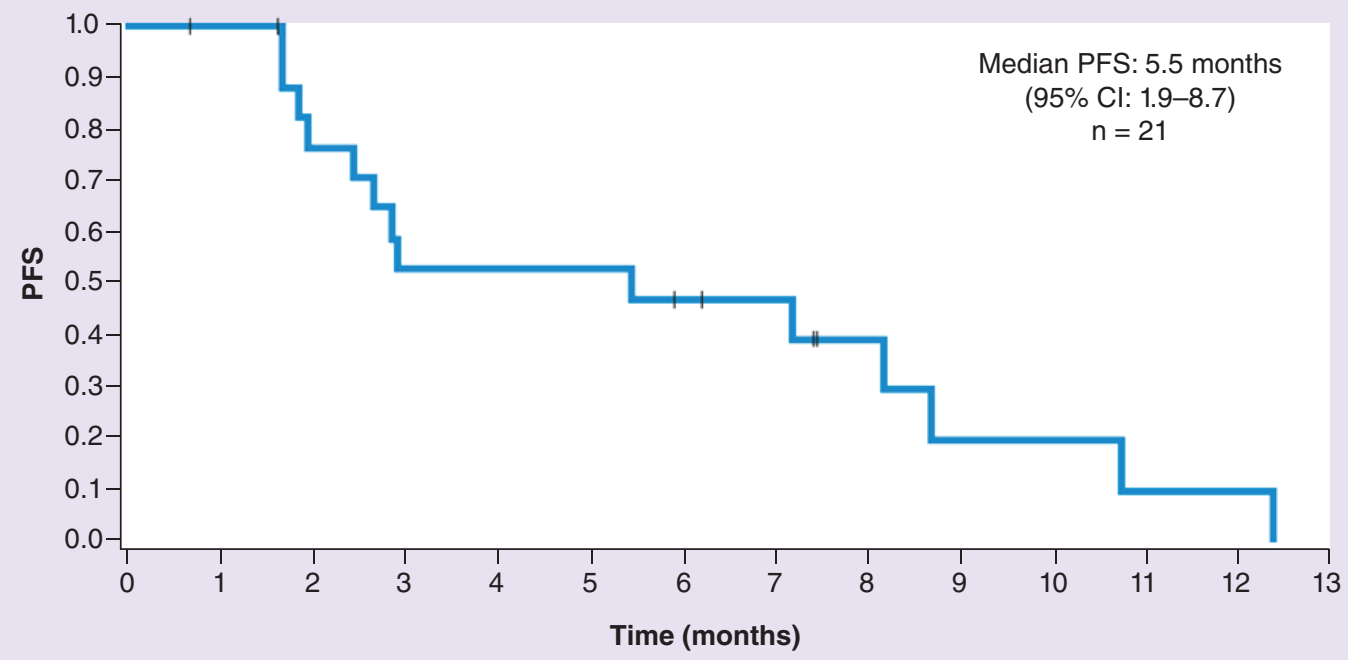

Figure 2. Progression-free survival.

PFS: Progression-free survival.

\section{Assessments}

The primary end point is OS rate 12 months after the start of treatment with nintedanib plus docetaxel. Secondary end points include progression-free survival (PFS) by investigator assessment (radiological progression), OS, objective response rate (ORR) and DCR. Response is assessed based on routine staging investigations and assessed in accordance with the Response Evaluation Criteria in Solid Tumors. Incidence and severity of adverse events (AEs) are being reported according to the National Cancer Institute Common Terminology Criteria for Adverse Events, version 4.03 .

\section{Statistical analyses}

All statistical analyses are descriptive. The PFS was calculated using the Kaplan-Meier method, from the start of nintedanib treatment to the date of death or disease progression, whichever occurred first.

\section{Results}

\section{Patient characteristics}

Baseline characteristics for the 22 patients included in this analysis are shown in Table 1 . In 17 patients for whom data were available, four had received pemetrexed maintenance therapy. In addition, 17 patients had an initial biopsy, showing that 15 (88\%) did not exhibit EGFR mutations or EML4-ALK translocations. The status of the remaining two patients is unknown. Of 11 patients for whom data were available, eight were PD-L1 positive.

\section{Efficacy}

At the time of this data snapshot (data cut-off: 1 August 2018), median duration of follow-up was 6.0 months (95\% CI: 3.5-8.8).

As shown in Table 2, response data were available for 12 patients; seven (58\%) had a PR and a further $3 / 12$ had stable disease, leading to an overall DCR of $83 \%$ in this patient population receiving later-line treatment. Response and DCRs with nintedanib plus docetaxel (58 and 83\%, respectively) were higher than those reported with second-line ICI therapy in the same patient population (23 and 38\%, respectively).

Median PFS (Figure 2) was 5.5 months (95\% CI: 1.9-8.7; n = 21). Fourteen PFS events had occurred; ten patients had disease progression and four patients had died. Seven patients were censored and data were not yet available for one patient. Treatment duration by treatment for each patient is shown in Figure 3. The OS data are not yet mature so are not reported. 


\begin{tabular}{|c|c|c|}
\hline \multicolumn{2}{|l|}{ Characteristics } & \multirow{3}{*}{$\begin{array}{l}\text { n (\%) } \\
58(45-76)\end{array}$} \\
\hline Median age, years (range) & & \\
\hline \multirow[t]{2}{*}{ Sex, n (\%) } & Male & \\
\hline & Female & $7(32)$ \\
\hline \multirow[t]{5}{*}{ ECOG PS, n (\%) } & 0 & $4(18)$ \\
\hline & 1 & $12(55)$ \\
\hline & 2 & $2(9)$ \\
\hline & 3 & 1 (5) \\
\hline & Missing & $3(14)$ \\
\hline \multirow[t]{5}{*}{ Tumor stage at baseline, $\mathrm{n}(\%)$} & IIIA & 1 (5) \\
\hline & IIIB & $3(14)$ \\
\hline & IV & $12(55)$ \\
\hline & Unknown & $3(14)$ \\
\hline & Missing & $3(14)$ \\
\hline \multirow[t]{2}{*}{ Brain metastases, n (\%) } & Yes & $4(18)$ \\
\hline & No & $18(82)$ \\
\hline \multirow[t]{5}{*}{ Previous first-line therapy, n (\%) } & Cisplatin or carboplatin + pemetrexed & $8(36)$ \\
\hline & Cisplatin + vinorelbine & 2 (9) \\
\hline & Carboplatin + paclitaxel & 1 (5) \\
\hline & Carboplatin + paclitaxel or docetaxel + bevacizumab & $2(9)$ \\
\hline & Carboplatin + vinorelbine + bevacizumab & 1 (5) \\
\hline \multicolumn{3}{|c|}{ Best response to first-line therapy, $\mathrm{n}(\%)$} \\
\hline & $C R$ & 0 \\
\hline & PR & $3 / 14(21)$ \\
\hline & SD & 6/14 (43) \\
\hline & PD & $5 / 14(36)$ \\
\hline & Not documented & 8 \\
\hline \multicolumn{3}{|l|}{ Previous second-line therapy, n (\%) } \\
\hline & Nivolumab & $17(77)$ \\
\hline & Pembrolizumab & $5(23)$ \\
\hline \multicolumn{3}{|c|}{ Best response to second-line therapy, $n(\%)$} \\
\hline & CR & 1/13 (8) \\
\hline & PR & $2 / 13(15)$ \\
\hline & SD & $2 / 13(15)$ \\
\hline & PD & $8 / 13(62)$ \\
\hline & Not documented & 9 \\
\hline
\end{tabular}

\section{Table 2. Best response to treatment with nintedanib plus docetaxel.}

\begin{tabular}{|ll}
\hline Response & $\mathrm{n} / \mathrm{N}(\%)$ \\
\hline CR & 0 \\
\hline PR & $7 / 12(58)$ \\
\hline SD & $3 / 12(25)$ \\
\hline PD & $2 / 12(17)$ \\
\hline ORR & $7 / 12(58)$ \\
\hline DCR & $10 / 12(83)$ \\
\hline
\end{tabular}

Response data were available for 12 patients at the data cut-off (1 August 2018).

CR: Complete response; DCR: Disease control rate; ORR: Objective response rate; PD: Progressive disease; PFS: Progression-free survival; PR: Partial response; SD: Stable disease. 


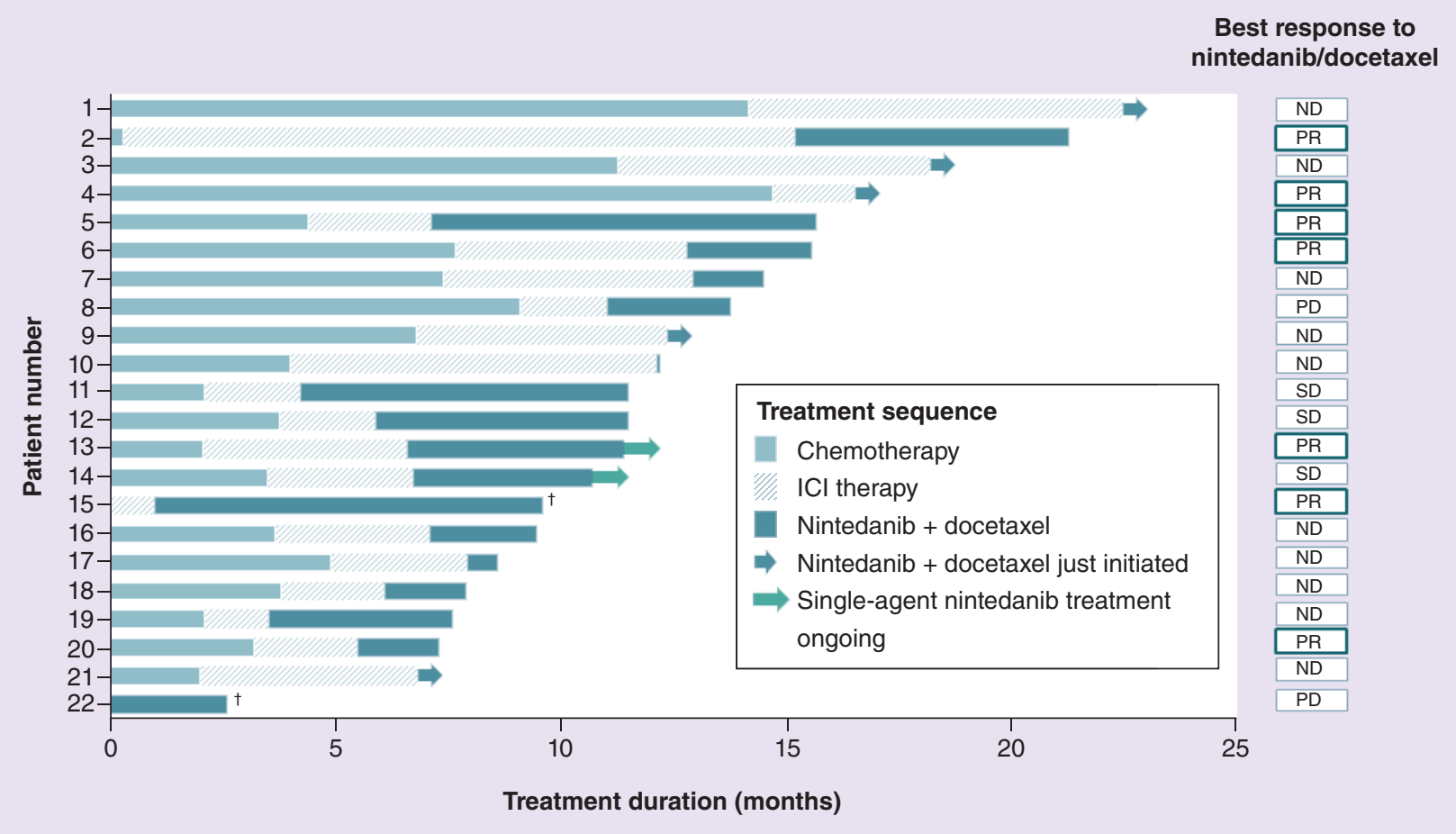

Figure 3. Swimmer plot showing treatment duration by treatment for each patient. Data cut-off: 1 August 2018. †Previous therapies not documented.

ICI: Immune checkpoint inhibitor; ND: Not documented; PD: Progressive disease; PR: Partial response; SD: Stable disease.

\section{Table 3. Treatment-related adverse events reported in $>10 \%$ of patients $(N=22)$.}

\begin{tabular}{|c|c|c|c|c|}
\hline \multirow[t]{2}{*}{ Adverse event } & \multicolumn{2}{|c|}{ Nintedanib related } & \multicolumn{2}{|c|}{ Docetaxel related } \\
\hline & All grades $n(\%)$ & Grade $\geq 3 \mathrm{n}(\%)$ & All grades $\mathrm{n}(\%)$ & Grade $\geq 3 \mathrm{n}(\%)$ \\
\hline Diarrhea & $8(36)$ & 0 & $6(27)$ & 0 \\
\hline Fatigue & $1(5)$ & 0 & $3(14)$ & 0 \\
\hline Deterioration in general physical health & $2(9)$ & 0 & $3(14)$ & $1(5)$ \\
\hline Nausea & $3(14)$ & $1(5)$ & $4(18)$ & $2(9)$ \\
\hline Stomatitis & $6(27)$ & $3(14)$ & $6(27)$ & $3(14)$ \\
\hline Decreased white blood cell count & $3(14)$ & $3(14)$ & $5(23)$ & $4(18)$ \\
\hline
\end{tabular}

Safety

Safety was evaluated in all 22 patients. About 21 patients (95\%) had AEs during study treatment, with 16 patients (73\%) experiencing drug-related AEs. The most common treatment-related AEs are shown in Table 3. Eleven patients (50\%) had serious AEs during study treatment, and seven patients (32\%) had serious drug-related AEs. Seven patients $(32 \%)$ had at least one nintedanib dose reduction and five patients $(23 \%)$ required at least one docetaxel dose reduction. AEs led to discontinuation of study treatment in seven (32\%) patients.

There were two deaths in this cohort that were attributed to serious AEs and were attributed to progression of underlying disease; these deaths were not reported as drug-related.

\section{Discussion}

The standard of care for patients with lung adenocarcinoma has advanced, with many patients now receiving ICIs upfront. Increasingly, clinicians have to decide on the optimal treatment sequence after patients progress on ICI \pm chemotherapy. However, there is a paucity of clinical evidence to help answer this pressing question. 
Although it is a prospective noninterventional study, a clear and major limitation of this study is the low number of patients. Another is that the data included in this report reflect clinical data as documented and available at the time of data cut-off for an interim analysis, with missing data for some patients. Furthermore, owing to the noninterventional nature of this study, no examinations (such as scans to determine disease status per Response Evaluation Criteria in Solid Tumors) were undertaken in addition to those that would usually have been performed at the site and for the patient in question. This likely contributed to the lack of recorded data on response to previous lines of treatment.

Despite these limitations, our dataset provides some insights into this patient population. Results show consistent evidence of the clinical benefit of nintedanib plus docetaxel in patients who have progressed on previous ICI therapy, across multiple outcomes: in terms of PFS, ORR and DCR. The ORR reported in this study, at $58 \%$, is higher than that reported in adenocarcinoma patients in the Phase III clinical trial, in which the ORR was 15/322 (4.7\%). Stable disease rates were 25 and $55.6 \%$ in each study, respectively [4]. It is also worth noting that three patients had an Eastern Cooperative Oncology Group performance status (ECOG PS) of 2-3, whereas patients in the Phase III clinical trial were required to have ECOG PS 0-1.

There were no new safety signals or unexpected toxicities during treatment with nintedanib plus docetaxel in this setting. Given that patients would be expected to have reduced tolerance as they progress through lines of therapy [10], the tolerability of third-line nintedanib plus docetaxel in this setting was encouraging.

The outcomes with antiangiogenic (nintedanib plus docetaxel) combination therapy in this cohort are consistent with reports that patients with advanced NSCLC who have progressed on immunotherapy appear to benefit from enhanced efficacy of subsequent chemotherapy [11-14]. Our dataset is also in line with data from another, smaller study of 11 patients who received later-line nintedanib plus docetaxel after chemotherapy and ICI therapy: in that study, treatment with nintedanib plus docetaxel was associated with an ORR of $36.5 \%$, a DCR of $81.8 \%$ and a median PFS of 3.2 months [8]. Based on the consistently high ORR and DCR in the two independent cohorts, we propose that the rational sequencing of antiangiogenic therapy with nintedanib plus docetaxel after ICIs may be a promising approach to treatment in this patient population, and warrants further investigation.

The mechanisms underlying the potential efficacy of this treatment approach remain to be further elucidated. Tumors secrete various immunosuppressive growth factors (e.g., VEGF) and tumor-derived factors increase the presence of immunosuppressive cells [6]. It is well established that there is considerable interplay between tumor immune suppression and vascular remodeling $[15,16]$. An immunosuppressive TME is closely linked with VEGFpromoted angiogenesis [6], and the abnormal vasculature resulting from tumor angiogenesis is likely to contribute to some tumors' resistance to ICIs. For example, tumor-induced aberrant, leaky vasculature can hamper the migration of effector $\mathrm{T}$ cells into the tumor [17]. These mechanisms would also prime the tumor for antiangiogenic therapy.

Targeting the TME in this way could, therefore, reinstate and even enhance the effect of immunotherapies. Antiangiogenic therapy can support normalization of vessels, decreasing hypoxia and also promote changes in the tumor endothelium, including an increase in T-cell adhesion and T-effector cell infiltration into the tumor [17].

We, therefore, hypothesize that an antiangiogenic treatment strategy involving inhibition of VEGF, as well as PDGF and fibroblast growth factor, could tip the balance toward an immunosupportive TME in an 'angioimmunogenic switch' (AIS). This AIS may be especially relevant if a tumor's immunogenic profile has undergone changes, in other words become more immunosuppressive, as a result of progression on ICI treatment.

This gives rise to the question: how does immune checkpoint blockade alter the tumor vasculature and the TME during tumor progression? Immune cells in the tumor, particularly in a hypoxic environment, can promote neovascularization [18]. Preclinical data suggest possible candidates for shared regulators of angiogenesis and immune suppression in tumors. For example, myeloid-derived suppressor cells are important regulators of the immune response that have also been implicated in promoting tumor angiogenesis, invasion and metastasis [19]. Reports suggest that the presence of myeloid-derived suppressor cells in the TME correlates with decreased efficacy of immunotherapies, including immune checkpoint therapy [19]. Another candidate is transforming growth factor $\beta$, a cytokine with a role in angiogenesis and immunosuppression [19]. Mapping of tumor immune and angiogenic markers during the course of treatment could help characterize the different mechanisms involved in a potential 'AIS'. Further research in this area may facilitate patient selection and optimal treatment sequencing in future.

\section{Conclusion}

With this in mind, the first step is to continue collecting clinical data, which is ongoing; patient recruitment in the VARGADO study has been extended. In the meantime, clinicians should take note of the clinical benefit 
reported with nintedanib plus docetaxel in patients with advanced adenocarcinoma NSCLC who have progressed after treatment with ICIs.

\section{Summary points}

- Immune checkpoint inhibitors (ICls) are a new standard of care in advanced nontargetable non-small-cell lung cancer (NSCLC), but the optimal treatment sequence after progression on $\mathrm{ICl} \pm$ chemotherapy remains to be established.

- Nintedanib is an oral triple angiokinase inhibitor that is approved in the European Union and other countries in combination with docetaxel, and has shown significant overall survival benefit for adenocarcinoma patients after first-line chemotherapy.

- VARGADO is a prospective, noninterventional study of nintedanib plus docetaxel after first-line chemotherapy in the routine clinical treatment of patients with locally advanced, metastatic or locally recurrent adenocarcinoma NSCLC.

- Here, we present initial efficacy data from VARGADO cohort B, in which 22 patients received nintedanib plus docetaxel after progression on prior chemotherapy followed by nivolumab $(n=17)$ or pembrolizumab $(n=5)$.

- Median progression-free survival with nintedanib plus docetaxel was 5.5 months (95\% Cl: 1.9-8.7). Of 12 patients, seven (58\%) had a partial response and three showed stable disease, leading to a disease control rate of $83 \%$.

- The response rate, disease control rate and progression-free survival with nintedanib plus docetaxel in adenocarcinoma NSCLC patients who progressed on previous ICl therapy highlight the potential clinical benefit of rational treatment sequencing with antiangiogenic therapy after ICls.

Financial \& competing interests disclosure

W Gleiber, C Losem, H Mueller-Huesmann, M Schulze, C Franke and N Basara have nothing to disclose. C Grohé has received sponsorship or research funding and payment or other financial remuneration from AbbVie, AstraZeneca, Bayer, Boehringer Ingelheim, Merck Sharp \& Dohme, Pfizer and F Hoffmann-La Roche, and payment or other financial remuneration from Lilly. S Haas has received, during the conduct of the study, personal fees and nonfinancial support from Boehringer Ingelheim and, outside of the submitted work, personal fees and nonfinancial support from Bristol-Myers Squibb R\&D, personal fees from Roche Pharma AG, Pfizer Pharma GmbH, Celgene GmbH and Sanofi-Aventis Deutschland GmbH, and nonfinancial support from Pharma Mar. J Atz and R Kaiser are employees of Boehringer Ingelheim and R Kaiser has a patent EP 2994125 issued. This study was sponsored by Boehringer Ingelheim. Boehringer Ingelheim was involved in the study design, in the collection, analyses and interpretation of the data, and in the writing of and decision to submit this paper for publication. The authors have no other relevant affiliations or financial involvement with any organization or entity with a financial interest in or financial conflict with the subject matter or materials discussed in the manuscript apart from those disclosed.

Medical writing assistance, supported financially by Boehringer Ingelheim, was provided by Syneos Health, UK, during the preparation of this manuscript.

\section{Congress presentations}

This work was presented at the European Society for Medical Oncology Immuno-Oncology Congress 2018, which was held in Geneva, Switzerland, 13-16 December 2018 (Grohé et al. Efficacy and safety of nintedanib and docetaxel in lung adenocarcinoma patients following treatment with immune checkpoint inhibitors: first interim results of the ongoing noninterventional VARGADO study [NCT02392455], Poster number 55P).

Open access

This work is licensed under the Attribution-NonCommercial-NoDerivatives 4.0 Unported License. To view a copy of this license, visit http://creativecommons.org/licenses/by-nc-nd/4.0/

\section{References}

Papers of special note have been highlighted as: $\bullet$ of interest; $\bullet \bullet$ of considerable interest

1. Hilberg F, Roth GJ, Krssak M et al. BIBF 1120: triple angiokinase inhibitor with sustained receptor blockade and good antitumor efficacy. Cancer Res. 68(12), 4774-4782 (2008).

2. Boehringer Ingelheim. Data on file. (2019).

3. Vargatef ${ }^{\circledR}$ Summary of Product Characteristics. www.ema.europa.eu/docs/en_GB/document_library/EPAR_-_Product_Information/human/002569/WC500179970.pdf 
4. Reck M, Kaiser R, Mellemgaard A et al. Docetaxel plus nintedanib versus docetaxel plus placebo in patients with previously treated non-small-cell lung cancer (LUME-Lung 1): a Phase III, double-blind, randomised controlled trial. Lancet Oncol. 15(2), 143-155 (2014).

-• Primary analysis of the Phase III LUME-Lung 1 trial, demonstrating significantly prolonged overall survival with nintedanib plus docetaxel compared with placebo plus docetaxel in patients with adenocarcinoma non-small-cell lung cancer (NSCLC) following progression after first-line chemotherapy.

5. Planchard D, Popat S, Kerr K et al. Metastatic non-small-cell lung cancer: ESMO Clinical Practice Guidelines for diagnosis, treatment and follow-up. Ann. Oncol. 29(Suppl. 4), iv192-iv237 (2018).

- Updated guidance from the European Society for Medical Oncology recommending immune checkpoint inhibitors with/without chemotherapy as front-line treatment for nontargetable advanced NSCLC.

6. Fukumura D, Kloepper J, Amoozgar Z, Duda DG, Jain RK. Enhancing cancer immunotherapy using antiangiogenics: opportunities and challenges. Nat. Rev. Clin. Oncol. 15(5), 325-340 (2018).

-. A review discussing the potential for antiangiogenic agents to normalize the tumor vasculature and help to overcome immunosuppression within the tumor microenvironment.

7. Molife C, Hess LM, Cui ZL et al. Sequential therapy with ramucirumab and/or checkpoint inhibitors for non-small-cell lung cancer in routine practice. Future Oncol. doi: 10.2217/fon-2018-0876 (2019) (Epub ahead of print).

- Comparative overall survival analysis of sequential therapy with ramucirumab and/or immune checkpoint inhibitors in the routine care management of patients with advanced NSCLC.

8. Corral J, Majem M, Rodriguez-Abreu D et al. Efficacy of nintedanib and docetaxel in patients with advanced lung adenocarcinoma treated with first-line chemotherapy and second-line immunotherapy in the nintedanib NPU program. Clin. Transl. Oncol. doi:10.1007/s12094-12019-02053-12097 (2019) (Epub ahead of print).

-• Retrospective evaluation of nintedanib plus docetaxel in 11 patients who received nintedanib as part of the named patient use programme following pretreatment with chemotherapy and immunotherapy.

9. Grohe $\mathrm{C}$, Ukena D, Lang $\mathrm{S}$ et al. Nintedanib plus docetaxel in routine clinical practice: VARGADO, a German prospective non-interventional study (NIS) reflecting routine treatment conditions in an evolving NSCLC treatment landscape. Ann. Oncol. 29(Suppl. 8), 1504TiP viii545 (2018).

10. Genestreti G, Grossi F, Genova C et al. Third- and further-line therapy in advanced non-small-cell lung cancer patients: an overview. Future Oncol. 10(13), 2081-2096 (2014).

11. Leger PD, Rothschild S, Castellanos E, Pillai RN, York SJ, Horn L. Response to salvage chemotherapy following exposure to immune checkpoint inhibitors in patients with non-small-cell lung cancer. J. Clin. Oncol. 35(15 Suppl.), 9084 (2017).

12. Schvartsman G, Peng SA, Bis G et al. Response rates to single-agent chemotherapy after exposure to immune checkpoint inhibitors in advanced non-small cell lung cancer. Lung Cancer 112, 90-95 (2017).

13. Park SE, Lee SH, Ahn JS, Ahn MJ, Park K, Sun JM. Increased response rates to salvage chemotherapy administered after PD-1/PD-L1 inhibitors in patients with non-small-cell lung cancer. J. Thorac. Oncol. 13(1), 106-111 (2018).

14. Grigg C, Reuland BD, Sacher AG, Yeh R, Rizvi NA, Shu CA. Clinical outcomes of patients with non-small cell lung cancer (NSCLC) receiving chemotherapy after immune checkpoint blockade. J. Clin. Oncol. 35(15 Suppl.), 9082 (2017).

15. Mortara L, Benest AV, Bates DO, Noonan DM. Can the co-dependence of the immune system and angiogenesis facilitate pharmacological targeting of tumors? Curr. Opin. Pharmacol. 35, 66-74 (2017).

-• A review of therapeutic strategies in oncology targeting the interplay between angiogenesis and the immune system.

16. Croci DO, Mendez-Huergo SP, Cerliani JP, Rabinovich GA. Immune-mediated and hypoxia-regulated programs: accomplices in resistance to antiangiogenic therapies. Handb. Exp. Pharmacol. 249, 31-61 (2018).

17. Van Der Woude LL, Gorris MAJ, Halilovic A, Figdor CG, De Vries IJM. Migrating into the tumor: a roadmap for T cells. Trends Cancer 3(11), 797-808 (2017).

18. Missiaen R, Mazzone M, Bergers G. The reciprocal function and regulation of tumor vessels and immune cells offers new therapeutic opportunities in cancer. Semin. Cancer Biol. 52(Pt 2), 107-116 (2018).

19. Sharma P, Hu-Lieskovan S, Wargo JA, Ribas A. Primary, adaptive, and acquired resistance to cancer immunotherapy. Cell 168(4), 707-723 (2017). 